\title{
NUMERICAL INVESTIGATION OF FANO RESONANCES IN METAMATERIALS WITH ELECTRIC ASYMMETRY
}

\author{
M. Rotaru, J. K. Sykulski \\ School of ECS, University of Southampton, Highfield, Southampton, SO17 1BJ, UK \\ mr@ecs.soton.ac.uk, jks@soton.ac.uk
}

Keywords: asymmetric resonance, Fano resonance, metamaterials.

\begin{abstract}
The excitation of high quality factor asymmetric Fanotype resonances on a double layer metafilm structure is investigated through numerical simulation. The paper demonstrates that it is possible to design simple structures capable to sustain a very high quality factor resonance by reducing their radiation losses. An equivalent circuit formed by two linearly coupled resonant RLC circuits is extracted in an attempt to explain the observed Fano resonance through classical circuit theory.
\end{abstract}

\section{Introduction}

Electromagnetic metamaterials and their potential applications have attracted significant attention from the scientific community in recent years. The research is driven by the opportunity to achieve electromagnetic properties that lead to new phenomena such as negative refraction, perfect magnetic surface or cloaking [1], which have no equivalents in natural world. Most of such 'exotic' electromagnetic response of metamaterials is due to their resonant behaviour; hence achieving a high-quality factor resonance is essential to obtain an efficient metamaterial based device. Typically, a metamaterial cell comprises a metallic structure - in which currents can be induced - and a supporting dielectric substrate. Depending on the induced current distribution in the conducting region, different resonance modes can be excited within these cells. Most of these resonant structures do not have a very high resonant quality factor because they suffer significant radiation losses. Moreover, due to their relatively small size with respect to the wavelength of operation, the metamaterial cell structures are not able to provide a large enough volume for the confinement of electromagnetic field necessary to support high quality factor resonances. However, recent theoretical analysis and experimental results have shown that high quality factor resonances can exist on small structures. These are mainly due to the existence of asymmetric profile resonances which belong to a larger class labelled as Fano resonances. The asymmetry comes from the close coexistence of resonant transmission and resonant reflection. Previous work has shown that a certain small structural (geometrical) asymmetry has to be introduced to the shape of their conducting elements to excite such resonances [2, 3]. In our prior work we have shown that similar effects can be achieved without breaking the geometrical symmetry of the resonator but instead by introducing an electrical asymmetry through a combination of substrates with different dielectric properties [4]. Making such structures is not easy as different dielectric materials have to be joined and assembled in a film or on a board on which the metal structures of the metamaterial cell are then deposited.

In this work the idea of electric asymmetry is further pursued and a novel type of metafilm is proposed to simplify the fabrication process. The new approach introduces an extra degree of freedom in the design of the metamaterial unit cell that offers further possibilities in tuning and improving the device.

\section{Electrical Asymmetric Resonator}

The metafilm considered in this work consists of identical sub-wavelength metallic inclusions on a dielectric substrate that are arranged in a periodic array. The metallic structures are symmetrically split rings with one half of the structure being on the top layer of the substrate and the second half on the second layer, as shown in Figure 1. The metallic split rings are assumed to be made of copper with a thickness of $35 \mu \mathrm{m}$. The outer radius of the ring is $6 \mathrm{~mm}$ and the inner radius is $5.2 \mathrm{~mm}$. The size of the fundamental cell that is repeated throughout this metafilm is $15 \times 15 \mathrm{~mm}$. This array is considered in our numerical study as infinitely long in $\mathrm{x}$ and $\mathrm{y}$ directions. The dielectric substrate is FR4 with the permittivity $\epsilon_{\mathrm{r}}=4.07$. The total thickness of the substrate is $3 \mathrm{~mm}$, with the second layer at a distance of $1.5 \mathrm{~mm}$ from the surface (Figure 2). Transmission and reflection of this thin metamaterial was obtained from electromagnetic simulation under normal incidence conditions.

\section{Results and Discussions}

The metamaterial structure was modelled using commercial software (CST Microwave Studio) with periodic boundary conditions applied to simulate an infinite array. The normal incident waves were forced through the definition of Floquet ports on the top and bottom boundaries of the 3D model (in the $z$ direction Figure $3-$ not shown). The solution used a frequency sweep between 3 and $15 \mathrm{GHz}$. The transmission and 
reflection properties of the structures of Figures 1 and 2 depend strongly on the polarization of the incident electromagnetic waves. For this configuration a very interesting phenomena is observed when the electric field of the incident electromagnetic wave is polarised in the $y$ direction.
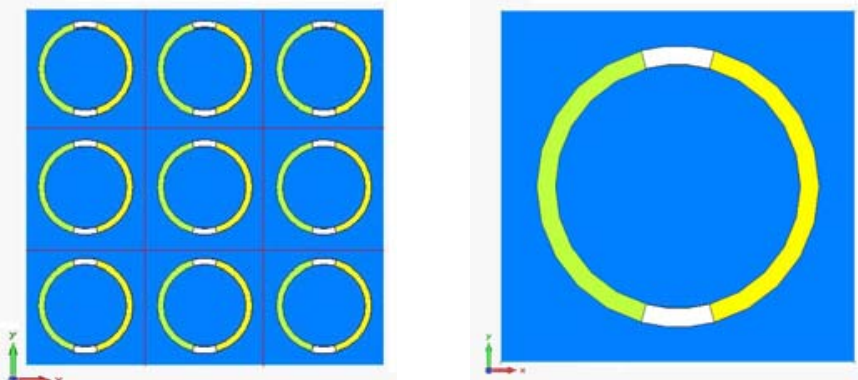

Figure 1. The metafilm structure (left) and the elementary cell structure (right).



Figure 2. A 3D view of the elementary unit cell.

The calculated results of reflection and transmission reveal an ultra sharp resonance at $5.3 \mathrm{GHz}$ (Figure 3 ). At this point (2 in Figure 3 ) the reflectivity loss is about $-4.6 \mathrm{~dB}$. There are two reflection peaks at $5.135 \mathrm{GHz}$ and $6.78 \mathrm{GHz}$ respectively. The origin of the unusually strong and narrow spectral response observed for this type of structures is due to the close
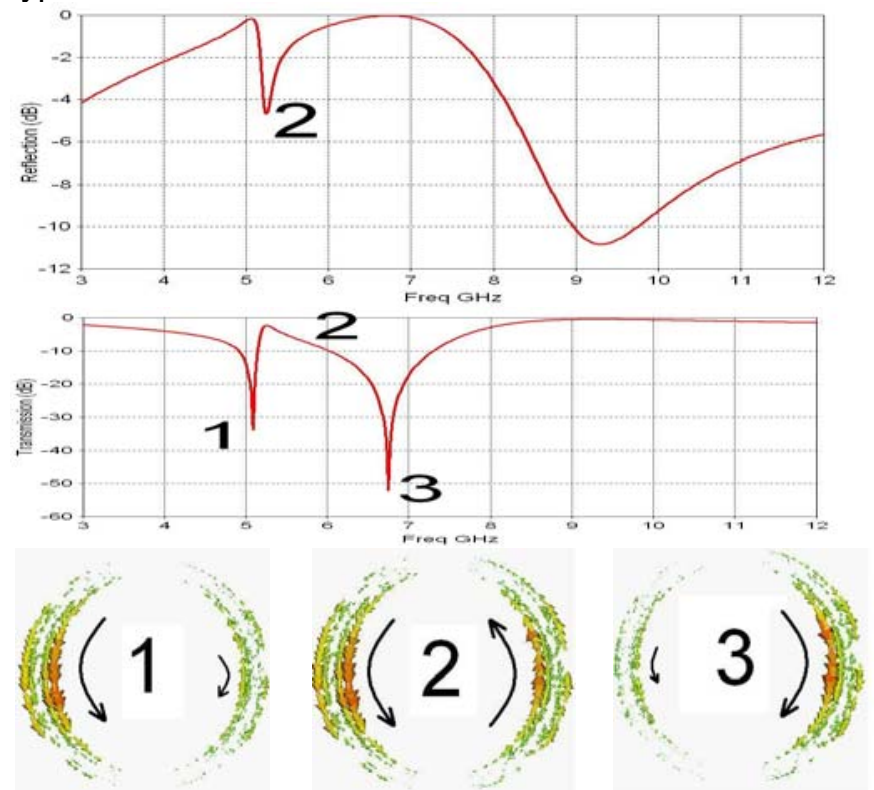

Figure 3. Normal incidence reflection and transmission and the corresponding current distributions for the three resonant modes. coexistence of a resonant transmission and a resonant reflection. The second resonant mode is a Fano resonance that is due to the interference of different scattering channels. In this case the induced currents on the two sides of the split ring are equal but $180^{\circ}$ out of phase, hence the fields produced by the two current will cancel each other creating this reflection resonance. On the other hand, the other two resonances are BreitWigner resonances because they are due to the interference of counter propagating waves in the same scattering channel. For these two modes one of the induced currents dominates the other one; consequently the structure will behave like a dipole at these frequencies. The dipole excited electromagnetic wave will interact with the incoming wave in a destructive manner producing a transmission resonance. The first type of resonance is inaccessible in structures without asymmetries - geometrical or electrical. The cell presented here has a certain electrical asymmetry due to the fact that one of the metal layers has a dielectric loading different to the other layer. This electrical asymmetry allows the weak coupling to free space, producing this Fano-like resonance.

\section{Conclusions}

A novel two layer metamaterial surface has been introduced. The metafilm can sustain very high quality resonances due to the electrical asymmetry created by the different dielectric loading of the two metallic parts. The existence of the Fano-type resonance is demonstrated through numerical simulation. The main advantage of the approach proposed here is the ease of fabrication of such structures as compared with the previous electrical asymmetric structures that needed two different dielectric materials to be combined in the same substrate.

\section{References}

[1] N. Engheta, R.W. Ziolkowski, "Metamaterials, physics and engineering exploration", IEEE Press John Wiley \& Sons, Inc, (2006).

[2] V.A. Fedotov, M. Rose, S.L. Prosvirin, N. Papasimakis and N.I. Zheludev, "Sharp trappedmode resonances in planar metamaterials with broken structural symmetry", Physical Review Letters, prl. 99, pp. 147401-1 - 147401-4, (2007).

[3] A. Ourir, R. Abdeddaim and J. de Rosny, "Tuneable trapped mode in symmetric resonator designed for metamaterials", Progress in Electromagnetics Research, PIER-101, pp. 115123, (2010).

[4] M. Rotaru and J.K. Sykulski, "Trapped Mode Resonances in Metalo-Dielectric Structures with Electrical Asymmetry", $21^{\text {th }}$ Symposium On Electromagnetic Phenomena in Nonlinear Circuits, Dortmund and Essen, Germany, pp. 11-12 29.0602.07, (2010). 